\title{
Information Technologies for Interoperability
}

\author{
J.P. Belaud ${ }^{1 *}$, D. Paen², L. Testard ${ }^{2}$ and D. Rahon ${ }^{3}$ \\ 1 Laboratoire de Génie chimique, CNRS UMR 5503, INPT-ENSIACET, 118, route de Narbonne, 31077 Toulouse Cedex 4 - France \\ 2 RSI, Parc technologique de Pré Milliet, 38330 Montbonnot - France \\ 3 Institut français du pétrole, Hélioparc Pau-Pyrénées, 2, avenue du Président Pierre Angot, 64000 Pau - France \\ e-mail: jeanpierre.belaud@ensiacet.fr - didier.paen@rsi-france.com - laurent.testard@rsi-france.com - daniel.rahon@ifp.fr \\ * To whom all correspondence can be addressed or duplicated
}

\begin{abstract}
Résumé - Technologies de l'information pour l'interopérabilité — Aujourd'hui, les systèmes d'information font largement appel aux réseaux. Le développement des applications informatiques complexes s'oriente vers un assemblage de composants disponibles sur un réseau local ou sur Internet. Ces composants doivent être localisés et identifiés en termes de services disponibles et de protocole de communication avant le lancement d'une requête. Cet article présente les principales technologies qui permettent à des solutions informatiques hétérogènes et réparties de collaborer. Le premier chapitre introduit les concepts de base des composants et des middleware. Les chapitres suivants décrivent les différents modèles de communication et d'interaction disponibles à ce jour et leur utilisation dans des applications industrielles. Enfin, le dernier chapitre montre comment des modèles différents peuvent interagir.
\end{abstract}

\begin{abstract}
Information Technologies for Interoperability - Information systems largely involve networking these days. The development of complex business applications is now focused on an assembly of components available on a local area network or on the net. These components must be localized and identified in terms of available services and communication protocol before any request. This article presents the most common technologies that allow heterogeneous and distributed software systems to collaborate. The first part of the article introduces the base concepts of components and middleware while the following sections describe the different up-to-date models of communication and interaction and their use in industrial applications. To finish, the last section shows how different models can themselves communicate.
\end{abstract}




\section{LIST OF ACRONYMS}

API Application Programming Interface

ASP Application Server Pages

B2B Business to Business

B2C Business to Consumer

BPEL Business Process Execution Language

BPEL4WS Business Process Execution Language for Web Services

BPML Business Process Markup Language

BPMI Business Process Management Initiative www.bpmi.org

CAPE Computer Aided Process Engineering

CASE Computer Aided Software Engineering

CCM CORBA Component Model

CLI Common Language Infrastructure

CLR Common Language Run-time

CML Chemical Markup Language www.xml-cml.org

CO CAPE-OPEN

COGents Agent-based Architecture for Numerical

Simulation www.cogents.org

CO-LaN CAPE-OPEN Laboratory Network www.colan.org

CORBA Common Object Request Broker Architecture

COTS Components Off The Shelves

(D)COM (Distributed) Common/Component Object Model

DCS

DLL

DTD

EAI

ebXML

EJB

ERP

GUI

GUID

HTML

HTTP

IDL

IIOP

IL

INDISS INDustrial and Integrated Simulation Software http://www.rsi-france.com/

IS Information System

ISO International Standard for Organization www.iso.org

IT Information Technologies

J2EE Java 2 Platform Enterprise Edition

JRMP Java Remote Method Protocol

JSP Java Server Pages

MDA Model Driven Architecture

MOM Message Oriented Middleware
OA Object Adapter

OASIS Organization for the Advancement of Structured Information Standards www.oasis-open.org

OLE Object Link Embedded

OMG Object Management Group www.omg.org

OO Object Oriented

OOP Object-Oriented Programming

OPC Object linking and embedding for Process

Control www.opcfoundation.org

ORB Object Request Broker

OS Operating System

PHP Hypertext Preprocessor

POSC Petrotechnical Open Standards Consortium www.posc.org

RFP Request For Proposal

RPC Remote Procedure Call

SAML Security Assertions Markup Language

SGML Standard Generalized Markup Language

SOA Service Oriented Architecture

SOAP Simple Object Access Protocol

SQL Structured Query Language

SVG Scalable Vector Graphics

UDDI Universal Description, Discovery, Integration

UML Unified Modeling Language

VB Visual Basic

W3C World Wide Web Consortium www.w3c.org

WSDL Web Services Description Language

WS-I Web Services Interoperability association www.ws-i.org

XMI XML Metadata Interchange

XML eXtensible Markup Language

XSL eXtensible Stylesheet Language

\section{INTRODUCTION}

It is a clear trend that enterprise software systems are becoming more and more complex. Applications have changed from simple stand-alone programs in a homogeneous environment to highly integrated and distributed systems in heterogeneous environments. Over the last ten years the need for web-enabled systems has led to additional requirements. In 1990's there was a need for connecting the whole software system of an enterprise, now the web environment results in the requirement to interconnect the software systems of enterprises to take advantage of the great business opportunities from the Net. The resulting Information software System (IS) plays a key role in enterprise; it is based on three fundamental parts: a set of data, a set of software processing and, a set of end-user presentation channels. Among usual needs for modern IS, the ability to interact and to exchange information with external or internal, homogeneous or heterogeneous and remote or nearby applications is a key point. 
The engineering field does not escape the problematic of information exchange. This journal issue has illustrated concrete examples of software interoperability for petroleum applications. As an example, Belaud (2001) introduced CAPE-OPEN (CO), a key technology for interoperability and integration of process engineering software components allowing a components off the shelves (COTS) engineering.

The need for integration has been illustrated in previous articles addressing interoperability issues in application domains. This final article focuses on the technologies for software interoperability with immediate industrial applications. IBM Glossary (2004) defines interoperability as the capability to communicate, execute programs, or transfer data among various software units. Information Technologies (IT) for interoperability are technologies that allow interoperability in a way that requires the final user to have little or no knowledge of the unique characteristics of those units.

The first section gives an overview of information systems and the associated technologies, then specific IT for communication, packaging and bridging are introduced.

\section{SOFTWARE ARCHITECTURE AND TECHNOLOGIES}

The different steps of software system development require one to view the system with respect to several individual perspectives such as those of end-users, analysts, designers, developers, etc. The software architecture is a good target as a candidate to manage these different points of view along the system lifecycle (Hofmeister et al., 2000). UML authors also recommend making use of a development process which is architecture-centric. According to Booch et al. (1998), software architecture encompasses the set of significant decisions about the organization of a software system such as:

- selection of the structural elements and their interfaces by which a system is composed, behavior as specified in collaborations among those elements;

- composition of these structural and behavioral elements into a larger subsystem and architectural style that guides this organization.

We can distinguish four steps in software system history: centralized architecture in 70's, decentralized architecture in 80 's, distributed architecture in 90's and web architecture in 2000 's. The latter integrates the web standard technologies and internet business. The use of web technologies has allowed more complex functionalities to be offered on the net; from information publishing to heterogeneous application integration. Services-Oriented Architecture (SOA), Model Driven Architecture (MDA), as well as grid, semantic, and autonomic architectures should be the main keywords for the next steps of software architecture.

As shown in Figure 1 web (enabled/distributed) architecture is based on multi-tier architecture that separates the presentation, business logic and data. We use this architectural vision to identify and place the different IT that can be selected for building the system (Serain, 2001). This approach concerns not only the physical view as shown in Figure 1 but also the logical view (e.g. code organization, application design, etc.).

- The presentation tier allows the graphical interaction with the end-users over the network using a thin client

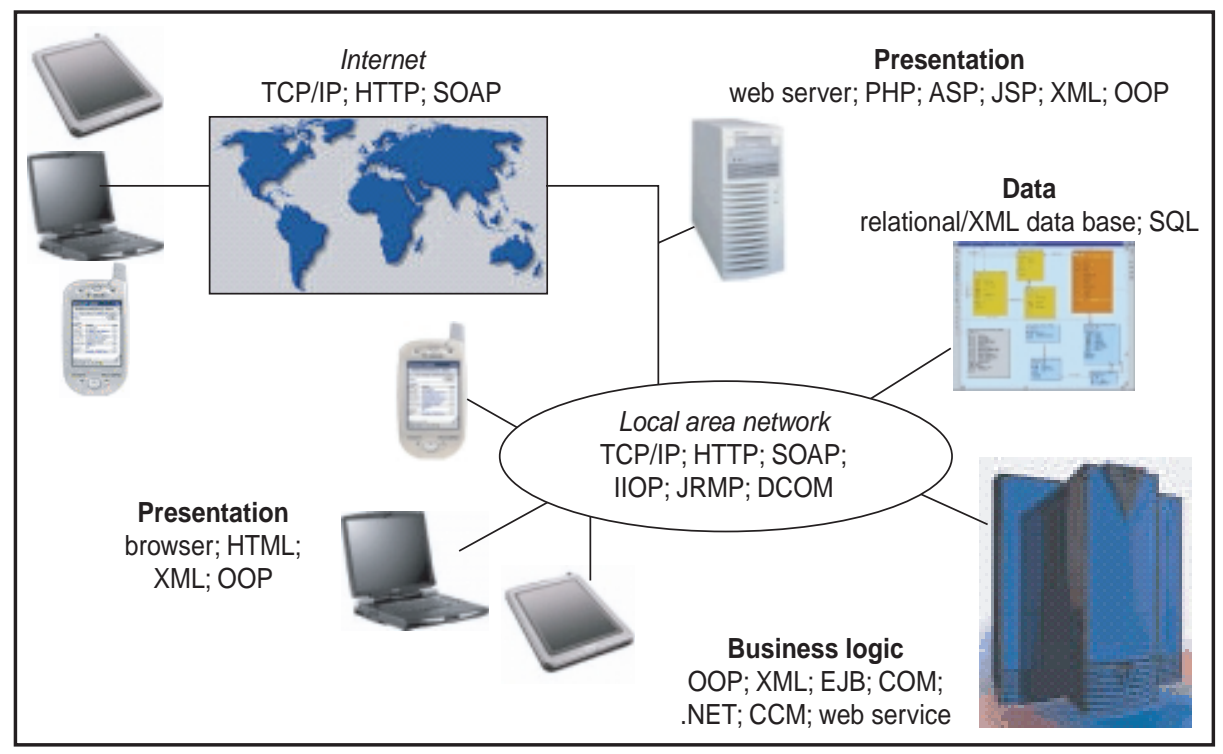

Figure 1

Web architecture basics. 
(basically the browser) or a rich client (a dedicated GUI). The thin client presentation is performed using web browser-HTML (with script languages and XML if any). The communication with the business logic tier is based on HTTP-TCP/IP. Web dynamic technologies such as PHP, Microsoft ASP and Java JSP do not differ from this principle since these pages are compiled and executed on the web server side to generate just-in-time HTML pages displaying the graphical interface of e-business or other applications. On the other side, the rich client presentation is developed with usual Object-Oriented Programming (OOP) languages such as Java, VB, C++, C\#, Delphi, etc., and the communication is carried out by protocols from middleware technology such as CORBA-IIOP, (D)COM, .NET Remoting, Java RMI-JRMP, XML-HTTP and SOAP according to component based programming. The middleware technology is the basic mechanism by which software components transparently make requests to and receive responses from each other on the same machine or across a network.

- The business logic tier encloses the application logic representing the enterprise know-how and rules. Usually this side is developed according to a component based approach with Unified Modeling Language (UML). This approach is based on advanced component models such as EJB from SUN/Java community, (D)COM and .NET from Microsoft, CORBA/CCM from OMG or web services from $\mathrm{W} 3 \mathrm{C}$. These components are mainly implemented following an $\mathrm{OO}$ programming and component intercommunication is performed by middleware inner protocols. The components run within a software framework called application server that provides a set of technical services such as transaction, identification, load balancing, security, data access, persistence, etc. The business logic tier can include legacy systems, web server and portal server.

- The data tier has the data persistence service with relational, XML and object data bases generally using SQL to manage data. In addition to usual data access techniques, work is being done on XML-enabled and web service data management (SQLXML).

To clarify the set of IT discussed in this article and introduced above using the architecture view, we propose to "classify" them according to six categories (the term technology is used in a broad sense):

- modeling technology: languages (UML), meta-model (MOF), model engineering (MDA);

- communication technology: Internet protocols (TCP/IP, HTTP), data model (XML), middleware and related communication protocol (CORBA-IIOP, DCOM, .NET Remoting, Java RMI-JRMP, SOAP);

- implementation technology: object-oriented programming (Java, C++, C\#, Eiffel), web programming (HTML, XML, ASP, JSP, PHP, PERL);
- packaging technology: component models and programming (EJB, COM, .NET, CCM, web services);

- bridging technology: COM-Java RMI, EJB-.NET;

- memory technology: relational, object, XML data base, SQL;

In order to develop such modern software applications and systems, technology selection involves many criteria. One main issue is to know if the technology is an (open) standard technology or proprietary technology. Open standard technologies are developed by software engineering from IT/software companies who collaborate within "neutral" organizations such as W3C, OASIS and OMG in accordance with a standardization process. Such organizations represent a new kind of actor additional to more traditional actors (academics, software-hardware-services suppliers and end-users companies). It is worth noting that this trend, issued from web philosophy, is also present in process and petroleum engineering field. Open standard technologies are freely distributed data models or software interfaces. They provide a basis for communication and common approaches and enable consistency (Fay, 2003), resulting in improvements in developments, investment levels and maintenance. Clearly the common effort to develop IT standard or domain oriented standard and its world-wide adoption by a community can be a source of cost reduction because not only is the development cost shared but also the investment is expected to be more future-proof. Open computing promises many benefits: flexibility/agility, integration capability, software editor independence, low development cost and adoption of technological innovation.

It is also possible to build IS from enterprise software especially for enterprise management. Enterprise Resource Planning (ERP), Enterprise Application Integration (EAI) and portal applications can provide build-in solutions that need to be tailored to enterprise context and requirements. These solutions, open source or commercial, involve standard technologies and compliant web architecture.

The following sections deal with information technologies for software interoperability explicitly communication, packaging and bridging technologies.

\section{COMMUNICATION TECHNOLOGY OVERVIEW}

\subsection{Interface, Class and Component}

An interface, a key element for middleware technology, is a collection of possible functions used to specify through its operations the services of a software unit. Depending on the selected middleware technology, interfaces are developed with a specific definition programming language such as OMG IDL for CORBA, Microsoft IDL for COM, Java interface for RMI and WSDL for web services.

A class is an object-oriented concept. It describes a set of shared objects and belongs to the implementation step. An 
object is an instance of a class. An object satisfies an interface if it can be specified as the target object in each potential request described by the interface. It belongs to the implementation step. However this object is distinct from the other usual objects since it collects the remote calls. The development of distributed software does not imply the choice of an actual object-oriented language (commonly $\mathrm{C}++, \mathrm{VB}$ and Java) since middlewares such as COM and CORBA introduce the notion of pseudo-objects.

The component is a software unit that encapsulates the implementation of business process logic. Sessions (2000) stresses the difference between component and object technology, the former being a packaging and distribution technology, focusing on what a component can do, while the latter is an implementation technology, focusing on how a component works. Objects and components are software entities; objects are typically fine grained units and interact in the same computing process while components are rather coarse grained units and are available outside their own process with respect to interface definitions. They are issued from different software design. The difference between these two states is clearly identified in the $\mathrm{CO}$ standard from CO-LaN. A CO compliant component is a piece of software that includes the supplier proprietary codes-objects or not—which realize or/and use $\mathrm{CO}$ interfaces. The communication between $\mathrm{CO}$ component instances is defined unambiguously by the $\mathrm{CO}$ interfaces introduced in Belaud and Pons (2002). In this case the middleware technologies are CORBA and COM.

\subsection{Middleware Principles}

Component based applications consist of several pieces of software which are executed independently and reside on the same host or on remote hosts over a network such as intraextra- Internet. There is a need for application integration and so for component communication through well defined interfaces. With this aim the middleware is a set of software that allows and organizes communication and information exchange between client component and server component. Figure 2 shows this technology as a universal communication bus, the "glue" of any IS, for integrating the different enterprise applications. It relies on a basic client-server communication model adding a key element; the interface defined in terms of Interface Definition Language (IDL) as defined previously.

A middleware solution provides mechanisms for interface definition and communication as well as additional services easing the use and implementation of component based software. The middleware interoperability protocol defines how the components communicate which each other. It defines marshalling process, how the data structures (integer, real, string, etc.) can be translated into network messages. There are three kinds of middleware technology: Message Oriented Middleware (MOM), Remote Procedure Call (RPC) such

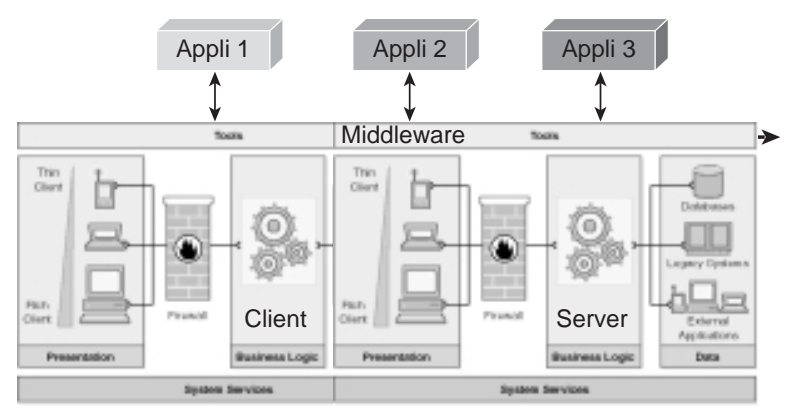

Figure 2

Middleware and client/server model.

as SOAP and Object-Oriented (OO). The interface design of $\mathrm{OO}$ middleware follows the object-oriented paradigm. At present the $\mathrm{OO}$ middleware solutions are (D)COM and .Net Remoting from Microsoft, CORBA from OMG and RMI from Java/SUN. COM, CORBA and SOAP are detailed in following sections.

All communication between software components is handled by middleware technology. Let us see the different alternatives for inter-system communication technologies e.g. how our system could process requests between software components. As a first approach we distinguish two ways for exchanging information: the data model and Application Programming Interface (API). These methods are usually used in IS based on open computing architecture. With the data model we can use point to point software integration and file format/database integration. But this static asynchronous communication is not appropriate to systems that use intensive integrated calculations. Indeed the performance penalty of managing physical files can be high and can prevent this approach being effective for exchanging information. Therefore interoperability can be achieved by API for carrying out inter-communication processes. We can identify basically two kinds of API technologies that are commonly used in any IS project, tightly-coupled and loosely-coupled middleware. Other distinctions can be used such as distinction based on data/control/presentation integration (Wassermann, 1990).

- Tightly-coupled middleware technology: this technology requests that software components have a close relationship. Typically this means that the components are built on identical middleware. OO middleware are typical examples. Here the components are closely linked by implementation dependence. For example a COM component can interoperate with a COM component on Windows. However non trivial solutions exist to break this tightly coupling such as bridging technologies discussed in Section 6. 
- Loosely-coupled middleware technology: software components do not need to share the common technology platform and they can be deployed easily over the web. The components are loosely-coupled by implementation independence. The web is based on this kind of protocol with HTML/HTTP. In this field the emerging industry standard for loosely-coupled inter-communication process is SOAP.

Section 3 introduces XML for information exchange by data model and Section 4 CORBA, COM and SOAP for information exchange by API.

\subsection{Marshalling}

An important notion for the API model is marshalling as remarked previously. Because distributed systems are heterogeneous (i.e. non uniform hardware, operating systems, etc.) the exchanges of data between different components must adhere to the same conventions with respect to the internal encoding of numeric data (little-endian, big-endian), to the encoding of data over a network (unicode strings).

Marshalling is the mechanism that ensures that the parameters of a method call are properly passed between the caller of the method and the callee (i.e. the code that implements the method).

\subsection{Implementation of Components}

The development of software that involves components is known as component based software engineering (Brown, 1996). This is special case of object oriented software engineering, and development techniques and methodologies apply here also. Object oriented software development methodologies exist and the implementation of software components benefit from the use of these methodologies e.g. unified process (Chan, 2003, discusses unified process applied to COTS), iterative models for components integration (Boehm, 2000).

In this paper, we address the particular problem of the implementation of software components. The main idea in components is to identify business objects that correspond to specialized activities, such as modeling, thermodynamics, numerical resolution, control and advanced control. Specialized engineers are developing new algorithms inside specialized components that implement interfaces dedicated to the corresponding domain. Each component can evolve independantly and remain compliant as long as it preserves the behavior of interfaces.

The use of UML (Booch et al., 1998) as a modeling language is the central point of software engineering tools. Some processes for software development employ standard

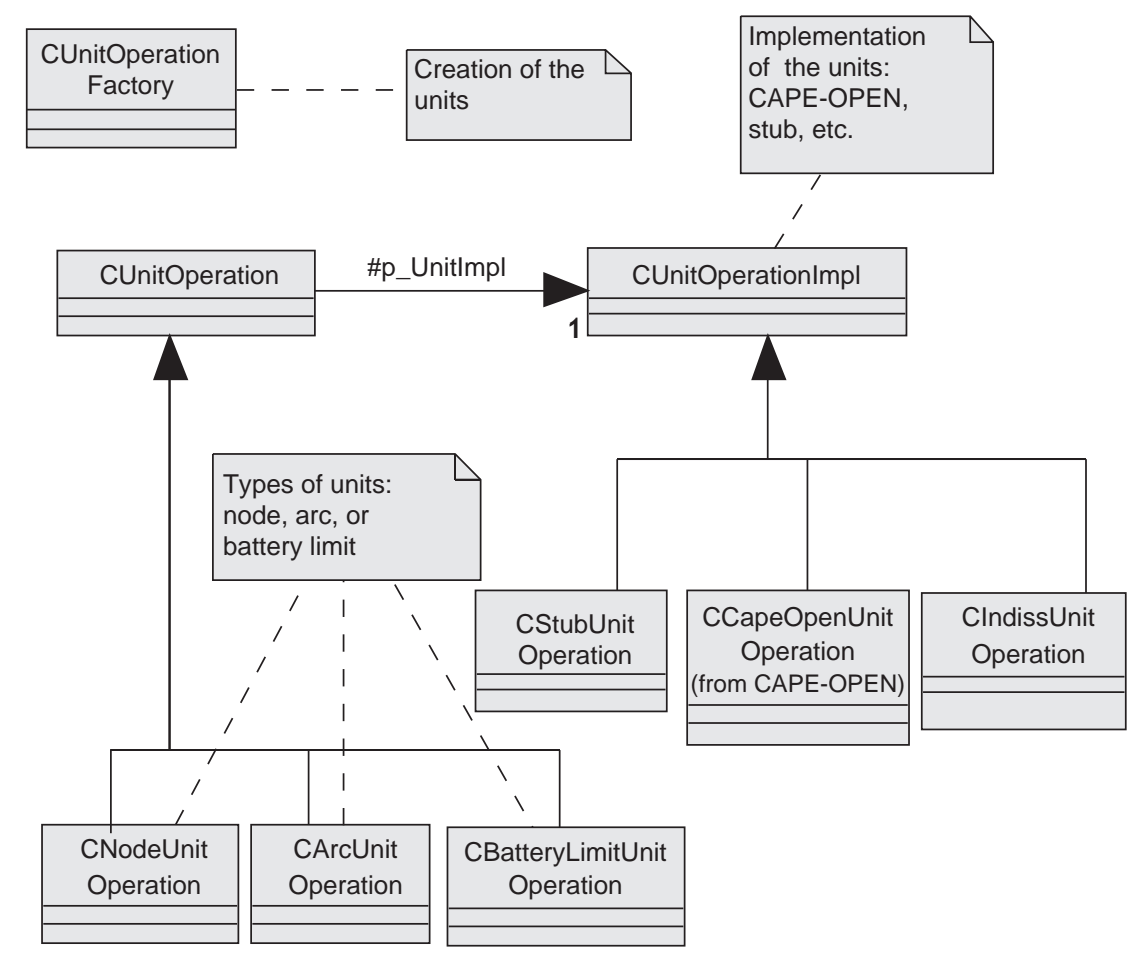

Figure 3

UML class diagram for unit operations.. 
use of UML and tools to develop software of high quality with an iterative lifecycle. UML use cases are good examples to describe project requirements. The analysis is here done using class diagrams with definition of high level classes, packages and interfaces for components. The developer has a global view of the classes and packages. Classes can implement different levels of complexity and heterogeneous environment. Figure 3 shows a class diagram from the INDISS project presented in Section 4.2. The dynamic behavior is modeled with sequential or collaboration diagrams. The development can be iterative due to the use of components. Some components are implemented very simply for first prototype; the idea is to attack risks early with an iterative lifecycle and to focus the process on the architecture first. It is better to detect major problems at the beginning rather than at the end of the project. Then releases are planned with evolving level of detail.

Application classes are developed by application team (numerical, thermodynamic, etc.) and technical classes are developed by IT team for communication, management of middleware data types and memory allocation. This separation is natural with UML class diagram. The use of middleware types and structures is very complex and has to be developed by a specialist IT team in specific technical classes (Fig. 4).

UML CASE (Computer Aided Software Engineering) tools enhance the capacity for changes in round-trip engineering. This is important to always have an up-to-date UML model. Then analysis and design documents can be generated from the model. Class source code is also generated from the model. The round-trip functions are essential for traceability quality requirements. The framework of a modern CASE tool is able to handle links with tools for software development:

- editor of source code;

- wizard of code environment, such as wizard to generate classes for Microsoft COM interfaces;

- configuration management tool.

Thus component based software development and UML modeling allow efficient cooperation of applicative and IT teams in an iterative lifecycle process.

\section{COMMUNICATION TECHNOLOGY BY DATA MODEL}

Inter-application communication by data model requires the definition of a standard data format, because the effective representation of the data is the heart of this model. In order to be adopted by major actors of application development, such a format should be standard, robust and open (i.e. can be easily tailored to specific business needs). The W3C consortium released the XML specification to address this problem.

\subsection{The XML Language}

EXtensible Markup Language (XML) is a simple, very flexible text format derived from SGML. Originally designed to meet the challenges of large-scale electronic publishing, $\mathrm{XML}$ from $\mathrm{W} 3 \mathrm{C}$ is also playing an increasingly important role in the exchange of a wide variety of data on the web and elsewhere.

XML is an extensible file format because it is not a fixed format like HTML (a single, predefined markup language). Instead, XML is actually a meta-language-a language for describing other languages-which can be specialized to meet the needs of a particular domain, like scientific or business domains (XMLFAQ, 2004). XML targets web development, due to its syntax being close to the syntax of HTML, thus providing natural transformations to produce web documents, but also targets other computer areas, such technical data handling, knowledge management, etc. Furthermore, the XML language itself is really easy to read and write, not only for specialized software tools but also for humans, which was not the case for SGML derived file formats.

XML files are based on UNICODE, which provides consistent representation whatever the language of the writer of the file and its reader. XML documents are generally made of elements, an element being enclosed between tags:

$<$ molecularWeight $>18.5<$ /molecularWeight $>$

An attribute is an additional property of a tag:

$<$ Element Type="Pure">

$<$ Name $>$ NITROGEN $<$ /Name>

$<$ Elements

Finally, elements can be nested, providing a hierarchical view of a data file:

$<$ MixtureDefinition>

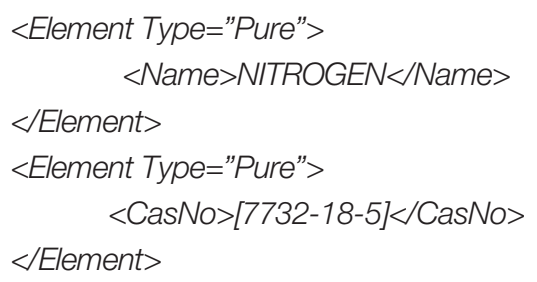

These simple language elements are the base of the syntax of XML files, although the language also covers notions such as namespaces, imports, typing, etc., to facilitate management of the data contained in these files.

A DTD (Document Type Definition) is a formal description in XML declaration syntax of a particular type of document. It sets out what names are to be used for the different types of element, where they may occur, and how they all fit together. DTD can be inline directly in an XML file, as well as being referenced as an external resource, that may be shared by different files. Given a DTD, the validity of a XML document can be directly enforced by the XML parser 


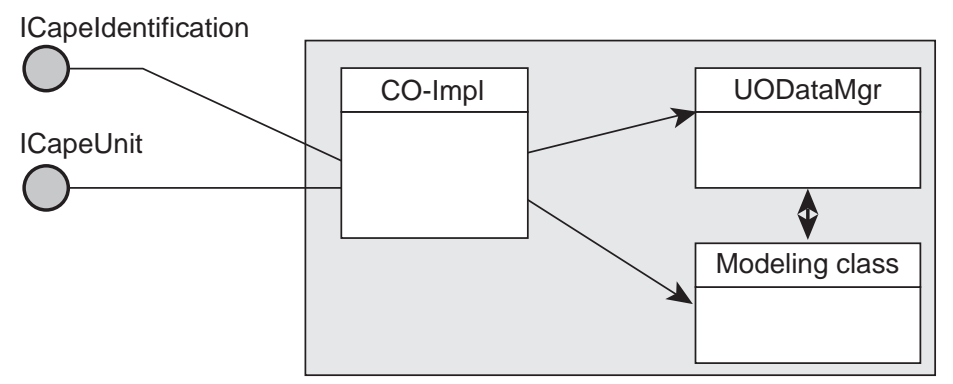

Figure 4

Domain and technical classes.

that reads the document, thus avoiding additional verifications of the document. An XML schema is another language that expresses syntax constraints on XML files, but instead of DTD, this language itself is based on a XML-like syntax.

XML files basically contain data along with enclosing tags describing the semantics of the data, these files can be processed in order to transform the structure of the file. XSL (eXtensible Stylesheet Language) aims at easy transformation of XML files into files of different format, XML compliant or not. XSL is a functional language that associates to the elements of the input XML files a set of transformations of the data contained in the input file.

One of the main use of XSL language is to transform XML files containing technical data, for example a list of data, into a more user friendly presentations, for example a table containing exactly one data per line, with associated color set depending on the nature of the data, and displayed on a standard internet browser. Figure 5 shows an typical architecture: a business application (a plant monitoring system, or a process simulation software) produces an XML compliant data file, which can be used by other, domain specific, applications (like a script that will extract all eventsrelated data in the file to produce an event $\log$ ), or by a generic tool (like a browser) which can produce a user friendly view of the data contained in the input file, with the help of business standard stylesheet.

\subsection{XML Specializations}

Since XML is a meta-language, it gave birth to other languages, which are dedicated to particular business domains. Amongst the many specializations of XML, we can enumerate:

- SVG is an XML sub language that is used nowadays to specify vector graphics and render them in commercial browsers.

- XMI from OMG is an XML language that can be used to describe UML entities, such as classes, diagrams, relationship, etc.

- CML is an XML sub language that describes the geometry of molecules.

- MathML from W3C is an XML language for mathematical expressions.

- OntoCAPE from the COGents project is an XML language for (Computer Aided Process Engineering) CAPE definition.

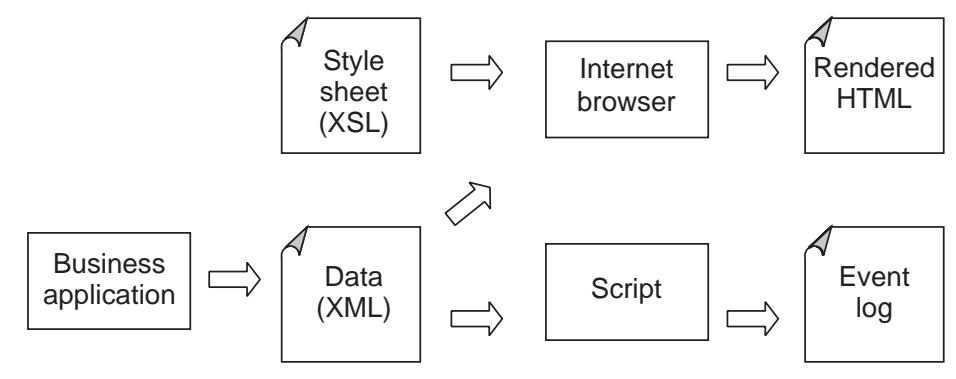

Figure 5

XSL transformation. 
- WellLogML: POSC, in conjunction with others in the oil industry, initiated a project in 1999 to design an XML schema for exchange of digital well log data. Version 1.0 of the WellLogML specification was published in April 2000 after extensive review by the oil and gas industry.

\subsection{Conclusion}

XML allows the flexible development of user-defined document types. It provides a robust, non-proprietary, persistent, and verifiable file format for the storage and transmission of text and data both on and off the web (XMLFAQ, 2004). It can be customized to meet the users' needs in many kinds of applications.

\section{COMMUNICATION TECHNOLOGY BY API}

Having seen the information exchange by data model, major technologies by API are presented and illustrated by short applications.

\subsection{OMG CORBA}

\subsubsection{OMG}

Dealing with heterogeneity in distributed computing enterprises is not easy. In particular, the development of software applications and components that support and make efficient use of heterogeneous networked systems is very challenging. Many programming interfaces and packages currently exist to help ease the burden of developing software for a single homogeneous platform. However, few help deal with the integration of separately-developed systems in a distributed heterogeneous environment. In recognition of these problems, the OMG was formed in 1989 to develop, adopt and promote standards for the development and deployment of applications in distributed heterogeneous environments. Since that time, the OMG has grown to become one of the larger software consortiums in the world, with approximately 800 members. These members contribute technology and ideas in response to Requests For Proposals (RFP) issued by the OMG. Through responses to these RFP, the OMG adopts commercially viable and supplier independent specifications for the software industry.

One of the first specifications to be adopted by the OMG was the CORBA specification. The last major update of the CORBA specification was in mid-2001 when the OMG released CORBA version 3.0.

\subsubsection{ORB}

CORBA defines a model that specifies interoperability between distributed objects on a network in a way that is transparent to the programmer. CORBA achieves this by defining ways for specifying the externally visible characteristics of a distributed object in a way that is implementationindependent.

This model is based on clients requesting the services from distributed objects or servers through a well-defined interface, by issuing requests to the objects in the form of events. The Object Request Broker (ORB) is in charge of delivering requests to objects and returns any responses.

Everything in the CORBA architecture depends on an ORB. The ORB acts as a central object bus over which each CORBA object interacts transparently with other CORBA objects located either locally or remotely. Each CORBA server object has an interface and exposes a set of methods. To request a service, a CORBA client acquires an object reference to a CORBA server object. The client can now make method calls on the object reference as if the CORBA server object resided in the client's address space. The ORB is responsible for finding a CORBA object's implementation, preparing it to receive requests, communicating requests to it, and carrying the reply back to the client. A CORBA object interacts with the ORB, either through the ORB interface or through an Object Adapter (OA).

\subsubsection{OA}

The OA serves as the glue between CORBA object implementations and the ORB itself. An object adapter is an object that adapts the interface of another object to the interface expected by a caller. In other words, it is an interposed object that uses delegation to allow a caller to invoke requests on an object even though the caller does not know that object's true interface. OA's represent another aspect of the effort to keep the ORB as simple as possible.

\subsubsection{IDL}

CORBA objects are accessed through the use of an interface. OMG's Interface Definition Language (IDL) is used to define interfaces, their attributes, methods, and parameters of those methods within the interface. OMG IDL is just a declarative language (see Fig. 6), not a programming language. As such, it is not directly used to implement distributed applications. Instead, language mappings determine how OMG IDL features are mapped to the facilities of a given programming language. The OMG has standardized language mappings for Java, C, C++, Smalltalk, Python and Ada.

IDL code is processed to generate stubs (on the client side) and skeletons (on the server side). Real operations are then implemented on the server side using inheritance or delegation. Client programs to the IDL-defined object use stub files, which provide communication through the ORB to object implementation. Figure 7 illustrates the respective stub and skeleton roles. 


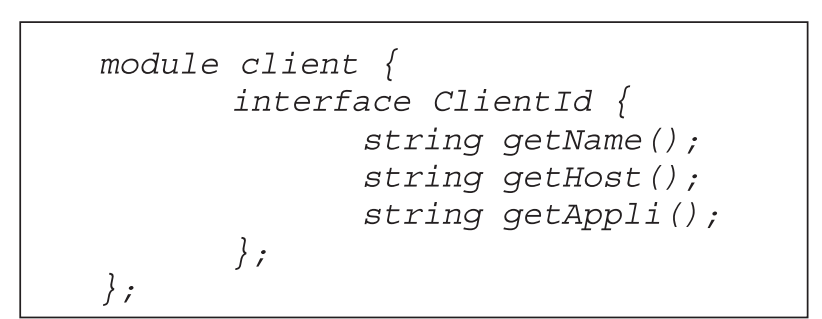

Figure 6

Short IDL example.

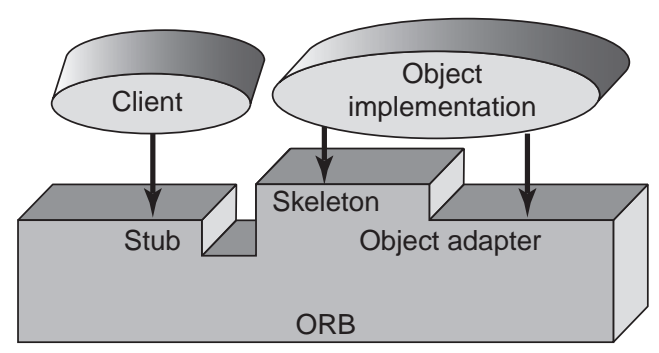

Figure 7

Comunication through ORB.

\subsubsection{IIOP and Services}

CORBA relies on a protocol called the Internet Inter-ORB Protocol (IIOP) for remote objects and CORBA defines a service-based architecture where distributed objects are only accessed through their interface without need for knowledge of the implementation details. Services can be shared by multiple clients and replaced by new ones supporting the same interface without disturbing client operations. The CORBAservices are standardized by the OMG in order to facilitate the development of applications and higher-level services across CORBA implementation. Among CORBAservices we can mention Naming, Lifecycle, Persistent, Transaction and Concurrency.

\subsubsection{Current Status}

Because CORBA is just a specification, it can be used on diverse platforms, including Mainframes, UNIX, Windows and so forth, as long as an ORB implementation exists for that platform. Currently, major ORB suppliers offer implementations for numerous platforms and programming languages. Numerous free implementations are also available for Java, C++ and Python. Puder (2004) maintains a comparative table of the different products available on the market.

\subsubsection{An industrial Product: OpenSpirit}

OpenSpirit (2004) is an industrial software platform based on the CORBA architecture that offers a standard access to multiple persistence solutions in the petroleum upstream domain. OpenSpirit allows independent applications to interoperate by sharing data and services. Through OpenSpirit, business applications can reach distributed data and dynamically share these data with the other connected applications, whatever the hardware, the programming language used or the software supplier. Given that integrated studies in the petroleum upstream domain require many applications from different vendors managing huge amounts of data, OpenSpirit allows end-users to significantly improve their business workflows. Petroleum engineers and geoscientists may integrate multivendor applications into a kind of "virtual application". Figure 8 shows the global architecture of the product.

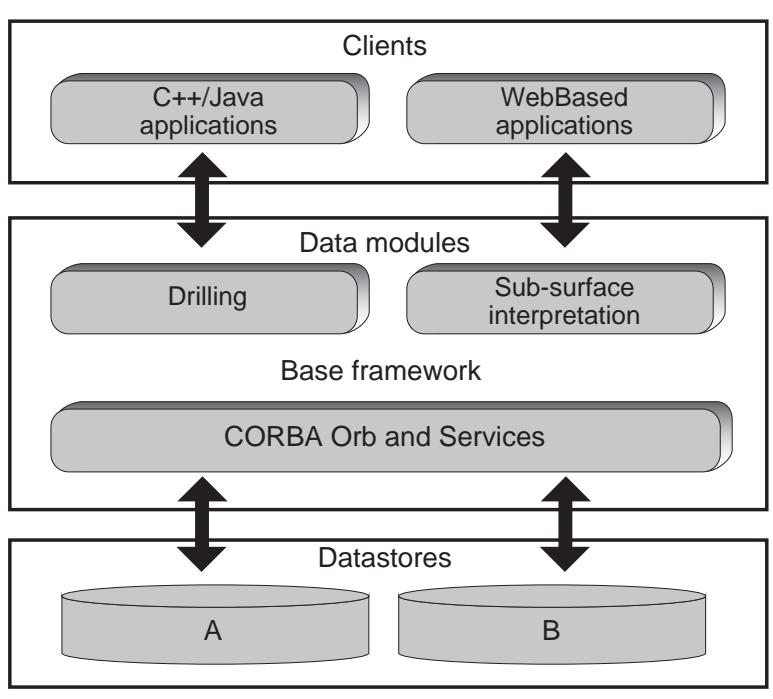

Figure 8

OpenSpirit architecture.

OpenSpirit is made up of:

- A Base Framework which offers a set of CORBA services and a set of specific objects (session, links, coordinate system, etc.). Figure 9 shows CORBA services in the OpenSpirit base framework.

- Data Modules which are domain specific (drilling, subsurface, seismic). Each one implements a set of standard objects relevant to that domain. One or more data servers are developed for each data module and each data server is specific to a particular physical project data store or corporate data repository. Data servers are responsible for managing data access between the data repository and the business objects.

\subsubsection{Conclusion}

CORBA is a mainstream information technology thanks to its capability to make software work together, regardless of where they are located or what language their are written in. It is now widely used even in traditionally conservative IT 


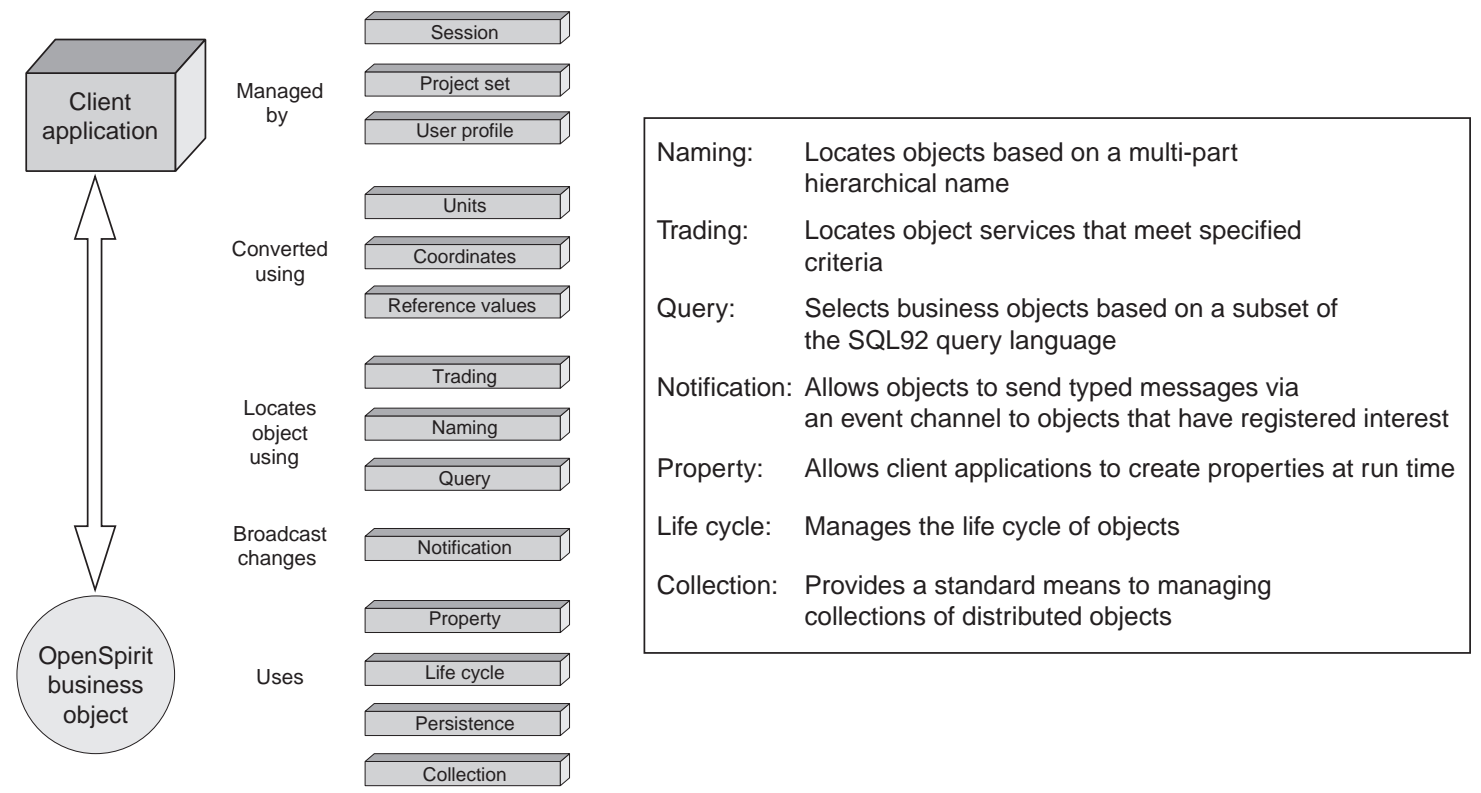

Figure 9

OpenSpirit CORBA services.

environments such as banking and insurance. Many commercial and free implementations of the standard are available for the main programming languages and CORBA is probably one of the best solutions to integrate legacy applications with new components based on recent technologies.

\subsection{Microsoft (D)COM}

Microsoft introduced the COM technology in its Windows platform in 1995, as a replacement for previous interapplication communication technologies (OLE, etc.). The first integration was performed in the NT family of Windows. The aim of the COM technology is to provide Windows developers a set of native OS functionalities as well as an object model in order for them to be able to develop component based application running on Windows. The term COM refers to Component Object Model (Box, 1998) and the term DCOM refers to Distributed COM (Eddon and Eddon, 1998): the former is the model of the components themselves, and the latter is the middleware that ties these components together.

\subsubsection{IDL and Interfaces}

Each COM object must be described by an IDL file whose syntax is very similar to the OMG standardized CORBA IDL syntax. The differences lie mainly in meta-data provided by Microsoft IDL syntax, such as the specification of a Global Unique Identifiers (GUID), the specification of additional semantics provided to the COM object, or handled types.
An IDL file can be compiled and the result is a code skeleton that can be used to implement a COM object, as well as other files than can be used to help the installation of the final executable on client computers.

An IDL can contain the declaration of the following elements:

- Types declarations: typedef, enums, etc.

- Interfaces declaration: methods, types, etc.

- Co-classes: a co-class is a set of interfaces that are simultaneously presented by a given component.

- Libraries: a library is a set of components that can be delivered together, as well as common type definitions, enumerated values, etc. A library is usually associated to a binary file (an executable file or a Dynamic Link Library), as a delivery medium of the components.

Microsoft provided several language mappings of the IDL syntax, namely mappings for $\mathrm{C}++, \mathrm{C} \#$ and VB.

\subsubsection{COM Objects}

A COM component is a binary file that contains the implementation of the methods of the interfaces it implements. Before being used by a client application, a COM component must be properly installed on a computer. The registration mechanism of a component depends on the nature of the executable it is contained in.

\subsubsection{COM Component Activation}

The process of creating a COM component and making it available to clients is sometimes referred to as component 


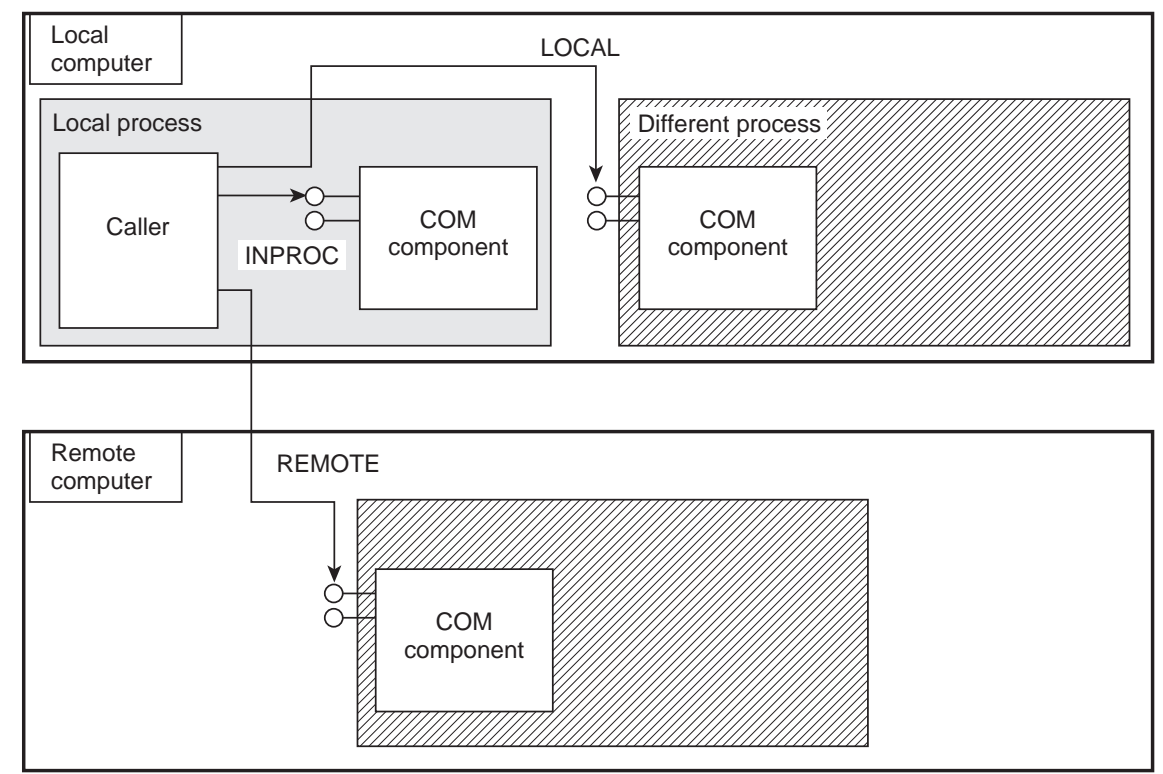

Figure 10

COM activation modes.

activation. Windows provides three ways to activate a component:

- Inproc: the component is loaded in the address space of the caller (i.e. the same process), and the method calls, to the methods of the interfaces of the component, are roughly the equivalent of usual function calls in $\mathrm{C}++$.

- Local: the component is loaded in a different address space than the process that requested the activation of the component. As a consequence the parameters of the methods must be properly packed before being sent and calling the method in the address space of the component.

- Remote: this activation mode is equivalent to the local mode, but the address space in which the component is loaded can be physically localized on another computer.

The three activations modes are shown the Figure 10 (the local process, e.g. the process of the caller is shaded in gray).

\subsubsection{IUnknown Interfaces}

Windows provides a special interface, the IUnknown interface that every COM object must implement. This interface is used for basically two kinds of operations:

- Interface navigation: given an interface implemented by a component, one can safely know if another interface is implemented by that component. This interrogation mechanism is fundamental for modern component based software engineering. In Figure 11, we show a synthetic view of a component that provides two interfaces: the interface IUnknown and another example interface IInterf. The hatched region corresponds to the implementation of the
IInterf interface, provided by the developer of the component, whereas the non hatched area corresponds to the system provided implementation of the IDefault interface. This default implementation provides the QueryInterface mechanism that enables the use of the implementation code of the interface IInterf (in the hatched area) from the "basic" IUnknown interface. Furthermore, this navigation mechanism is safe, i.e. at run-time, one can safely (without any risk of crash of the application, as opposed to the $\mathrm{C}$ language operation of cast) know whether a component supports or not a given interface.

- Reference counting: since no client of a given component is able to determine if an object must be present for future method calls, each client must adhere to a reference counting mechanism so that the OS is the only requester of effective object destruction.

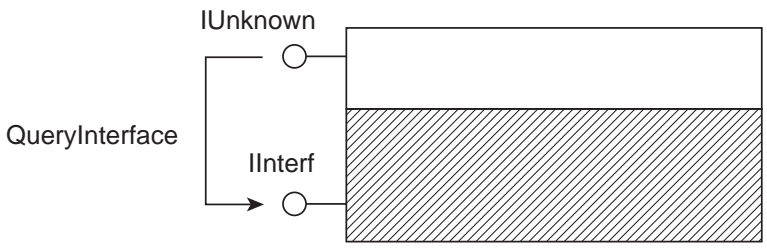

Figure 11

Interface navigation. 


\subsubsection{IDispatch Interfaces}

One other special interface provided by Windows is the IDispatch interface which is aimed specifically at Microsoft applications interoperability by being natively used by the VB language. This interface provides a mechanism called late binding, which in a word enables "textual calls" to be performed on a object (e.g "call the method M on the object"), the OS being responsible for effectively calling the method on the object or rejecting the call if the object does not support the interface or if the method is not part of the interface.

This mechanism is the basis of VB/COM interoperability. Since VB is interfaced with practically every Microsoft application (Excel, Access, Word) the IDispatch interface is one of the key technologies for Windows system integration of third parties software applications.

\subsubsection{Marshalling}

The standard behavior of this mechanism depends on the type of the parameter that have to be marshalled:

- The standard IDL types are handled naturally (standard RPC mechanism, the values are encoded before "being sent over the wire", Birrel and Nelson, 1984).

- UNICODE strings: the necessary memory allocations/ desallocations are performed by the OS.

- VARIANT: a VARIANT is a data structure that virtually encapsulates every IDL supported data type. The necessary memory allocations/desallocations are performed by the OS during the marshalling of such values.

- Interface pointers: interface pointers can be marshalled, thus enabling the remote use of an interface pointer from a remote computer (call back mechanisms).

\subsubsection{Operating System Related Considerations}

One important feature of COM is to be deeply integrated in Windows. This integration lies essentially in two areas: the use of OS resources and the fact that COM objects are handled by many Windows mechanisms, namely system administration and security.

COM mechanisms make extensive use of OS resources, namely:

- the system registry, used to perform the registration of COM objects;

- activation mechanisms;

- some global objects useful to use specific instances of COM objects (Running Object Table, Global Interface Table).

The integration of COM components as standard system objects enables one to apply security policies to these objects.

\subsubsection{Conclusion}

The COM object model is only suited to the development of components under Windows, and is often opposed to
CORBA as an object model for component based applications. While the close integration in Windows can be a serious advantage, mainly due to the sharing of system objects and to the fact that it requires no additional hazardous installation on the computers where it is used, it also has some disadvantages:

- COM is committed to Windows.

- COM is hard to administrate because of the inner complexity of OS related concepts.

- The life span of the COM technology is only dependent on Microsoft's willingness, because Microsoft is the only actor able to decide strategic choices concerning their component technology.

Before beginning the implementation of a component based application, the question of the choice of the correct component technology must be solved. The previous elements should be taken into account before proceeding to the final choice.

\subsubsection{An Industrial Solution: INDISS}

The INDISS simulation platform (INDustrial Integrated Simulation Software) is designed to provide all the necessary software tools that facilitate the implementation of applications in process engineering. One single core base will support various simulation environments that address different modelling and simulation needs thus avoiding duplication, additional engineering efforts and result discrepancies. Robustness and fidelity is achieved through the use of physical properties and reaction kinetics. INDISS is the result of an object-oriented development over ten years. The software is coded in $\mathrm{C}++$ under Windows environment. The open nature of its architecture is visible in several functionalities:

- Development of unit operations is independent from INDISS software. They can be converted into dynamic libraries and then loaded by INDISS.

- INDISS proposes its internal thermodynamic package, and an external thermodynamic package is also available to provide thermodynamic calculations in specific conditions.

- INDISS provides a GUI builder. This tool is used to build a customer interface based on a standard simulator.

The use of technologies that allow modularity and evolutivity is an essential point for INDISS development. Communication with Microsoft tools and software engineering effort in component based development are central. As an example, $\mathrm{CO}$ interfaces are integrated on the first level of design of INDISS components.

INDISS takes advantage of being developed under Microsoft tools by providing several levels of Microsoft interfaces. This can be done by using first versions of Microsoft interoperability tools. INDISS is a server that presents OLE (Object Link Embedded) interfaces. These interfaces allow simulation monitoring and access in reading and 
writing mode to each variable of INDISS unit operations. It is useful to automate test sequences and provide automatic reports. This link is easy to establish under Excel or with Visual Basic.

As described in the presentation of INDISS, development of unit operations is independent from INDISS software to produced libraries. This kind of library can only be loaded inside INDISS because it uses proprietary structures for data interfaces that are linked with the INDISS executable. COM, as a middleware, adds one level of indirection between INDISS and the units. The interface is described in an IDL file that ensures the compatibility of client and server. The middleware allows also by default a link with a local or remote server. The interface is fully described in a text file. The ease with which one can describe the interaction between client and server is an important point in defining business standards, as detailed in the next paragraphs.

\section{INDISS and DCS connection}

INDISS is a tool to build process engineering simulators : it is often necessary to connect INDISS to third party software like databases or control simulators. The traditional way to connect INDISS to a control simulator for a Distributed Control Systems (DCS) is to develop a new piece of software. The proprietary API of the DCS provider is used to exchange values of control and process values.

To avoid such developments, OPC is an emerging software standard designed to provide automation applications with easy access to industrial plant floor data. OPC means initially OLE for Process Control. OLE has since been restructured and renamed to ActiveX and then COM. The goal of OPC is to define a standard interface based on COM technology that allows greater interoperability between automation and control applications; control devices; and business and office applications.

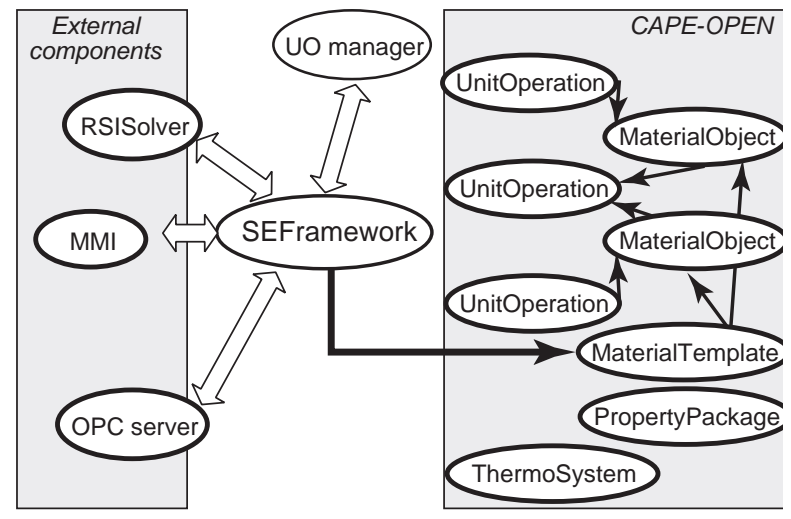

Figure 12

Components architecture.

\section{INDISS and CAPE-OPEN}

Based on CO requirements, INDISS is a component based multi layer client server architecture. As a result, INDISS is extremely flexible and facilitates the integration of third party components. The internal thermodynamic routines have been separated from the core platform and have been made available as standalone components. A third party can easily plug in its own thermodynamic or $\mathrm{CO}$ thermodynamic property packages without going through heavy engineering when coding is necessary (Fig. 12).

This increased flexibility is of extreme importance as it primarily benefits the customer. At this present stage all the tests to integrate and operate unit operations in a dynamic mode are positive and promising and future developments will further emphasize component technology and the use of $\mathrm{CO}$ interfaces.

\section{Conclusion on INDISS}

The capability of INDISS to interact with other software was a basis requirement. The middleware development has increased this need by providing business components. INDISS now provides an OPC link. RSI has tested the CAPE-OPEN interface to include an external thermodynamic server inside INDISS and is developing the CAPEOPEN interface for dynamic unit operations with other CAPE software providers. It is expected that in the next years, customers will be able to choose the software components that best match their need.

\subsection{SOAP and XML-Based Middleware}

HTML-HTTP act as loosely-coupled middleware technology between the web client (browser) and the business logic layer (web server). HTTP can be viewed as a simplified RPC middleware. Around the year 2000, Microsoft and IBM proposed using the XML data format over the internet protocols: HTTP — as transport layer - and XML—as encoding format -now constitute the key underlying technologies for web services. Web services are discussed in Section 5.4.

Many companies perform remote function calls by transferring XML messages on HTTP without standardized technology such as the XML-RPC specification from UserLand (2004). XML-RPC is designed to be as simple as possible, while allowing complex data structures to be transmitted, processed and returned. A set of compatible XML-RPC implementations that cover all operating systems and programming languages for Perl, Python, Java, C/C++, PHP, .NET, etc., are available. For example, Meerkat (2000) extends its open API with XML-RPC, affording a XMLbased interface to its aggregated database.

However the technology resulting from XML-HTTP most in vogue is currently SOAP (Simple Object Access Protocol) from W3C. This promising protocol is for application interoperability (components and web services) and is not directly 


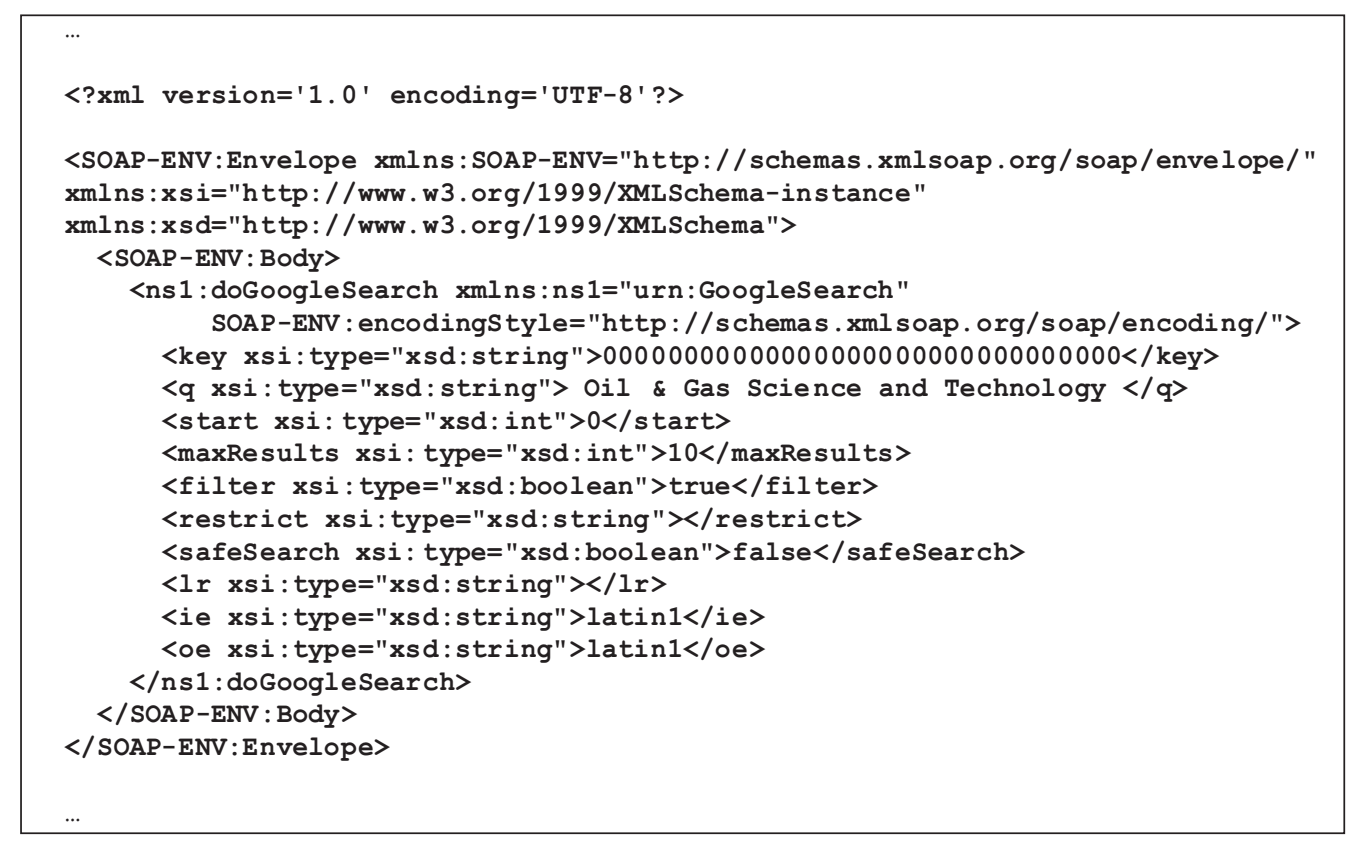

Figure 13

SOAP request messge sample.

related to an object-oriented view as its name could lead one to believe. SOAP (currently in version 1.2) was delivered in June 2003, as a lightweight protocol for exchange of information in a decentralized and distributed environment. It is an XML based protocol that consists in three parts: an envelope that defines a framework for describing what is in a message and how to process it, a set of encoding rules for expressing instances of application-defined datatypes, and a convention for representing remote procedure calls and responses. SOAP can handle both the synchronous request/response pattern of RPC architectures and the asynchronous messages of messaging architectures. Figure 13 presents a SOAP request message in a synchronous manner from Google Web APIs beta (2004). A SOAP request is sent as a HTTP POST. The XML content consists in three main parts (Glass, 2001):

- the envelope defines the namespaces used;

- the header is an optional element for handling supplementary information such as authentification, transactions, etc.;

- the body performs the RPC call, detailing the method name, its arguments and service target. In the example doGoogleSearch is the method name and key, q, start, maxResults, etc. are the arguments.

\section{Conclusion}

Whereas CORBA, RMI, (D)COM and .NET Remoting try to adapt to the web, SOAP middleware ensures a native connectivity with it since it builds on HTTP, SMTP and FTP and exploits the XML web-friendly data format. Reasons noted for the success of SOAP are its native web architecture compliancy, its modular design, its "simplicity", its text-based model (in contrast to binary and not self-describing CORBA, RMI, (D)COM, .NET protocols), its error handling mechanism, its suitability for being the common message handling layer of web services, its standardization process and its support from major software editors.

\section{PACKAGING TECHNOLOGY}

Middleware components run within a controlled runtime environment provided by the server editor. This packaging technology deals with the creation, management and destruction of the business component. With this technology the component developer no longer needs to write "technical" code that handles transactional behavior, security, database connection pooling, etc. because the architecture delegates this task to the server supplier. These techniques are now widely used in network solutions and this section describes the different technologies.

\subsection{EJB, Java Community Technology}

\subsubsection{Enterprise JavaBeans}

Enterprise JavaBean (EJB) proposes a high-level approach for building distributed systems. It allows application 
developers to concentrate on programming only the business logic, while removing the need to write all the common code required for any multi-tier application development scenario. For example, the EJB developer no longer needs to write code that handles transactional behavior, security, connection pooling or threading.

In essence, EJB is a server component model for Java and is a specification for creating server-side, scalable, transactional, multi-user, and secure enterprise-level applications. EJB can be deployed on top of existing transaction processing systems including traditional transaction processing monitors, web servers, database servers, application servers.

\subsubsection{EJB Architecture}

EJB are based on three key concepts:

- Framework: a business component resides in a container that provides a technical contract (naming, security, transaction, persistence, pooling, destruction, etc.). The EJB should sign a contract with the container (implements an interface) to take advantage of the container contract (Fig. 14).

- Proxy: a client never accesses instances of the enterprise bean's classes directly. It uses the enterprise bean's remote interface. Remote call to the EJB services should be encoded on the client side and decoded on the server side. This is realized through stub and skeleton proxies.

- Factory: an enterprise bean's instance cannot be created and removed directly. These functions are devoted to a factory called home interface.

\subsubsection{Types of Enterprise Bean}

Three types of enterprise bean are available, each corresponding to a particular business logic.

- Stateless and stateful session beans: conversational beans. They cannot persist and are not shared between clients.

- Entity beans: represents legacy data. They persist in a data store and can be shared by different clients.

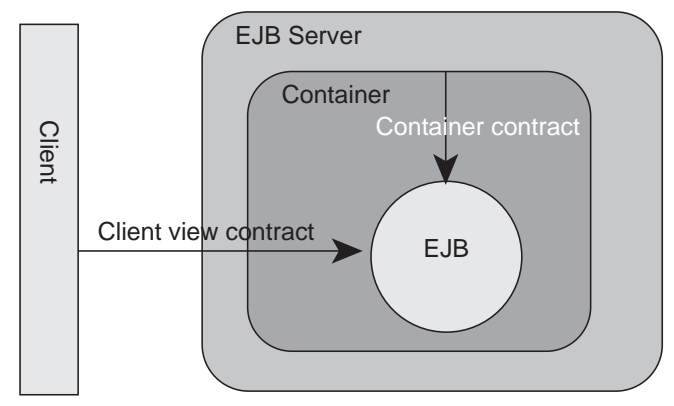

Figure 14

EJB Container.
- Message-driven beans: they can send and receive messages asynchronously. Clients do not access to a messagedriven bean.

\subsubsection{Benefits of Using EJB}

In multi-tier architecture, it does not matter where the business logic is. With EJB, business logic can reside on any server, while adding additional tiers if necessary. The EJB's containing the business logic are platform-independent and can be moved to a different, more scalable platform if necessary. An EJB can move from one platform to the other without "any" change in the business-logic code. A major highlight of the EJB specification is the support for ready-made components. This enables one to "plug and work" with offthe-shelf EJB's without having to develop or test them or to have any knowledge of their inner workings.

\subsubsection{EJB Communication}

RMI is used as communication protocol for EJB clients. However, EJB may provide CORBA/IIOP protocol for a robust transport mechanism and pure CORBA clients can access EJB as EJB clients. Currently, a highlight of OMG's CORBAServices is the wide range of features they provide to an enterprise application developer. In the future, rather than trying to rewrite these services, EJB server vendors may simply wrap them with a simplified API, so EJB developers can use them without being CORBA experts.

A complete description of EJB technology can be found in Gopalan (2004).

\subsubsection{Java 2 Platform, Enterprise Edition (J2EE)}

The Java 2 Platform, Enterprise Edition (J2EE) is a set of coordinated specifications and practices that together enable solutions for developing, deploying, and managing multi-tier server-centric applications. Building on the Java 2 Platform, Standard Edition (J2SE) the J2EE platform adds the capabilities necessary to provide a complete, stable, secure, and fast Java platform to the enterprise level. It significantly reduces the cost and complexity of developing and deploying multitier solutions.

Enterprise Java Beans are part of the J2EE platform but the platform provides also many other key technologies, for example complete web services support.

\section{2 .NET, Microsoft Technology}

The Microsoft response to J2EE is called .NET, and provides a set of standard components, languages, etc., aimed at the development of business applications. The different elements are:

- The CLR (Common Language Run-time) is the mechanism that allows every compliant language to interoperate closely (objects defined in one language can be used in 
another one). Languages such as C\#, VB.NET, can be compiled "on the fly" into Intermediate Language (IL): the resulting code is called managed code because the IL provides services and concepts that help the execution of such code (for instance, garbage collecting to prevent memory leaks, sandboxing to prevent malicious code being executed, etc.). On the other hand, CLR also enables the execution of unmanaged code, letting the global security policy of the virtual machine decide if it can be allowed. The CLR is the equivalent of Java virtual machine, the IL of the Java byte code.

- .NET common classes are a set of common classes that are provided by the framework and that ease the enterprise application development. These classes are dedicated to management of files and should act as a replacement for Microsoft foundation classes.

- ASP .NET provides classes used during Active Server Pages (ASP) creation, enabling the execution of C\# code within HTML pages.

- WinForms is the technology used to create graphical applications.

- .NET remoting is the .NET middleware technology that handles the deployment of distributed applications in NET (Browning, 2002). Holloway (2002) compares it to web services.

The .NET and J2EE architectures are very similar, each having its advantages, and its respective defaults. The key technology is the programming language, i.e. $\mathrm{C \#}$ for Microsoft .NET and Java for EJB. These object oriented languages are similar in scope (simplified OO languages), run on virtual machines and thus are naturally portable on different architectures (Windows CE, XP for .NET, all Unix flavors for Java). As a starting point, Farley (2000) proposes a comparison of J2EE and .NET and TMC (2002) has revisited the "famous" pet store benchmark that compares the SUN J2EE Pet Store and the Microsoft.NET Pet Shop.

\subsection{CCM, OMG Technology}

CORBA Component Model (CCM) is a specification that focuses on the strength of CORBA as a server-side object model. It concentrates on issues that must be addressed to provide a complete server side middleware component model. It can be described as a cross-platform, crosslanguage superset of EJB. The CCM gives developers the ability to quickly build web-enabled enterprise scale applications while leveraging the industrial strength of CORBA. Tight integration with EJB leverages CORBA's crossplatform and multiple-language capabilities.

The CCM is part of the CORBA 3.0 specification. It extends the CORBA object model by defining features and services in a standard environment that enable application developers to implement, manage, configure and deploy components that integrate with commonly used CORBA services. These server-side services include transactions, security, persistence and events. An open source implementation of a CCM platform, OpenCCM, is developed by the ObjectWeb consortium (2004).

\subsection{Web Services, W3C Technology}

Web technologies are more and more used for application-toapplication communication as explained in previous sections. At first, software suppliers and IT experts promised this interconnected world thanks to the technology of web services. Web services propose a new paradigm for distributed computing (Bloomberg, 2001) and are one of today's most advanced application integration solutions (Linthicum, 2003). They help business applications to contact a service broker, to find and to integrate the service from the selected service provider.

However, even if the idea of web services has generated too many promises, web services should be viewed for now as a part of a global enterprise software solution and not as a global technical solution. In a project, web services can be used within a general architecture relying on Java EJB or on Microsoft's .NET framework. Newcomer (2002) gives keys for selecting between J2EE and .NET with respect to web services support.

Many projects already utilize web services, sometimes with non standard technologies, particularly for non critical intranet applications. Even if web services lack advanced functionalities, many advantages like lower integration costs, the re-use of legacy applications, the associated standardisation processes and web connectivity can plead in favor of this new concept for software interoperability and integration (Manes, 2003).

\subsubsection{Definition}

A web service is a standardised concept of function invocation relying on web protocols, independent of any technological platform (operating system, application server, programming language, data base and component model). BearingPoint et al. (2003) focus on the evolution from software components to web services and write: "a web service is an autonomous and modular application component, whose interfaces can be published, sought and called through Internet open standards". We see the introduction of web services as a move from component architectures towards Internet awareness, this context implying the use of associated technologies i.e. HTTP and XML, and an e-business economic model. Current component technology based on EJB, .NET and CCM being not fully suitable, web services provide a new component approach for providing functionality anywhere, anytime and to any device. 


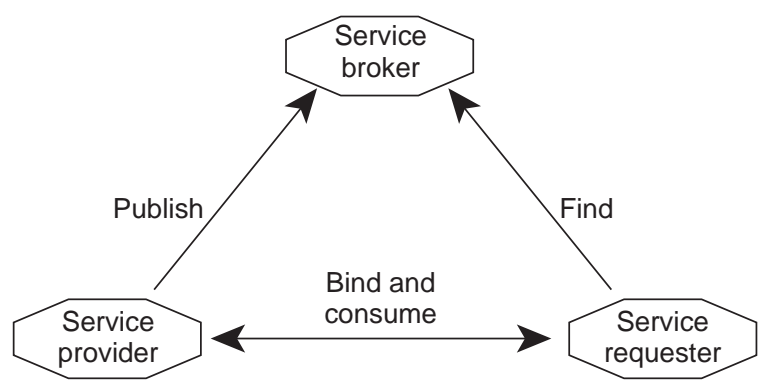

Figure 15

Key principles of web services.

\subsubsection{Key Principles}

IBM and Microsofts' initial view of web services, first published in 2000, identified three kinds of roles (Fig. 15):

- a service provider publishes the availability of its services and responds to requests to use its services;

- a service broker registers and categorizes published service providers and offers search capabilities;

- a service requester uses service brokers to find a needed service and then employs that service.

These three roles make use of proposed standard technologies: UDDI (Universal Description Discovery Integration) from the OASIS consortium, WSDL (Web Services Description Language) and SOAP (Simple Object Access Protocol) from the W3C. UDDI acts as a directory of available services and service providers; WSDL is a XML vocabulary to describe service interfaces (similar to IDL function).

Further domain specific technologies related to web services are being developed, e.g. the following ones proposed by the OASIS consortium, a consortium of companies interested in the development of e-business standards:

- ebXML is a global framework for e-business data exchange;

- BPEL (formerly BPEL4WS) is a proposed standard for the management and execution of business processes based on web services;

- SAML aims at exchanging authentication and authorization information;

- WS-Reliable Messaging is for ensuring reliable message delivery for web services;

- WS-Security aims at forming the necessary technical foundation for higher-level security services.

Additionally standards from RosettaNet and BPML (Business Process Markup Language) from BPMI deal with the management of business processes.

Simply stated, the interface of a web service is documented in a file written in WSDL and the data transmission is carried out through HTTP with SOAP. SOAP can also be used to query UDDI for services. The functions defined within the interface can be implemented with any programming language and be deployed on any platform. In fact, any function can become a web service if it can handle XML-based calls. The interoperability of web services is "similar" to distributed architectures based on OO middleware such as CORBA, RMI or (D)COM but web services offer a loose coupling, a non intrusive link between the provider and the requester, due to the loosely-coupled SOAP middleware. Bloomberg (2001) compares these different architectures.

Oellermann (2002) discusses the creation of enterprise web services with real business value. Basically he reminds us that a web service must provide the user with a service and needs to offer a business value. The technically faultless but closed .NET "my services" project from Microsoft demonstrates that is always challenging to convince final users. With Google web APIs beta (2004), software developers can query the Google search engine using the web services technology (search, cache and spelling services). Figure 16 shows the search service part from WSDL description and Figure 17 illustrates the C\# source code of service requester application written for Microsoft .NET platform. In this example, there is no interaction with a UDDI server.

With so many advantages for integration and interoperability one could expect a massive adoption by software solutions architects. However the deployment of web services still remains limited. In addition to technical issues, three main reasons can be noted:

- Web services are associated to SOAP, WSDL and UDDI. The UDDI directory of web services launched in 2000 by IBM, Microsoft, Ariba, HP, Oracle, BEA and SAP, was operational at the end of 2001 with three functions (white, yellow and green pages). However due to technical and commercial reasons this worldwide repository that meets an initial need (to allow occasional, interactive and direct interoperability) founded on the euphoria of e-business years does not match the requirements of enterprise systems. Entrusted to OASIS in 2002, UDDI version 3 proposes improvements in particular for intranet applications.

- The simplicity and interoperability claimed by web services are not so obvious. Different versions of SOAP and incompatibilities of editors' implementations are source of difficulties, to such a degree that editors created the WS-I consortium to check implementations of standards of web services across platforms, applications, and programming languages.

- The concept was initially supported by a small group of editors (with Microsoft and IBM leading); now the "standards battle" (BEA, IBM and Microsoft from one side and Iona, Oracle and Sun from the other side) and the multiplication of proposed standards are weakening the message of web services (Koch, 2003). 


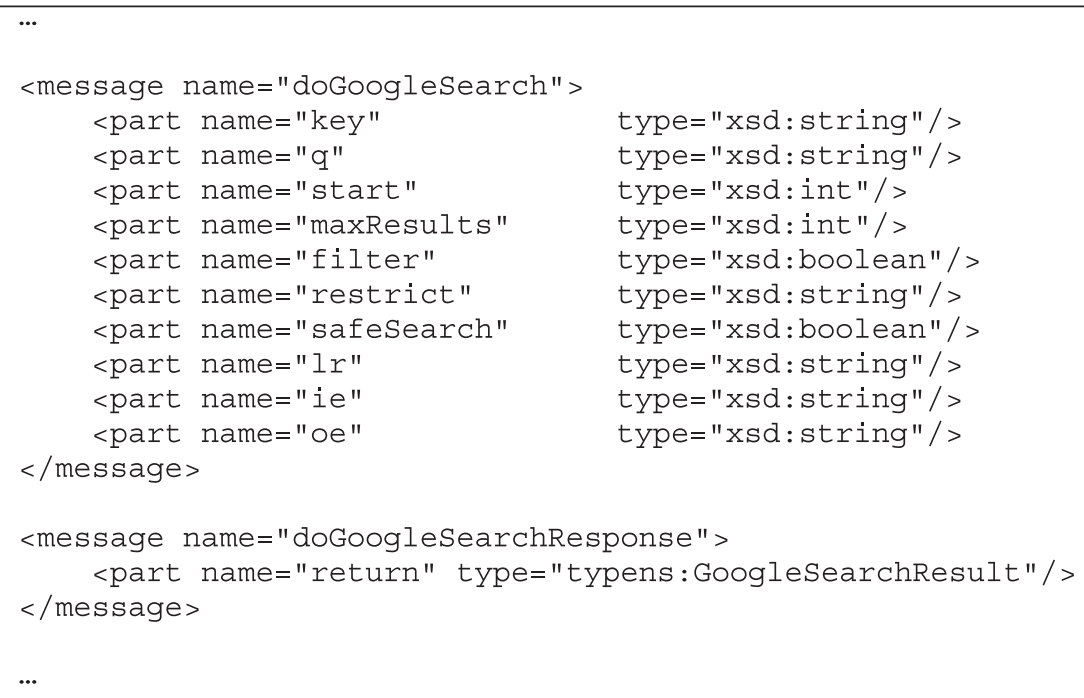

Figure 16

WSDL sample.

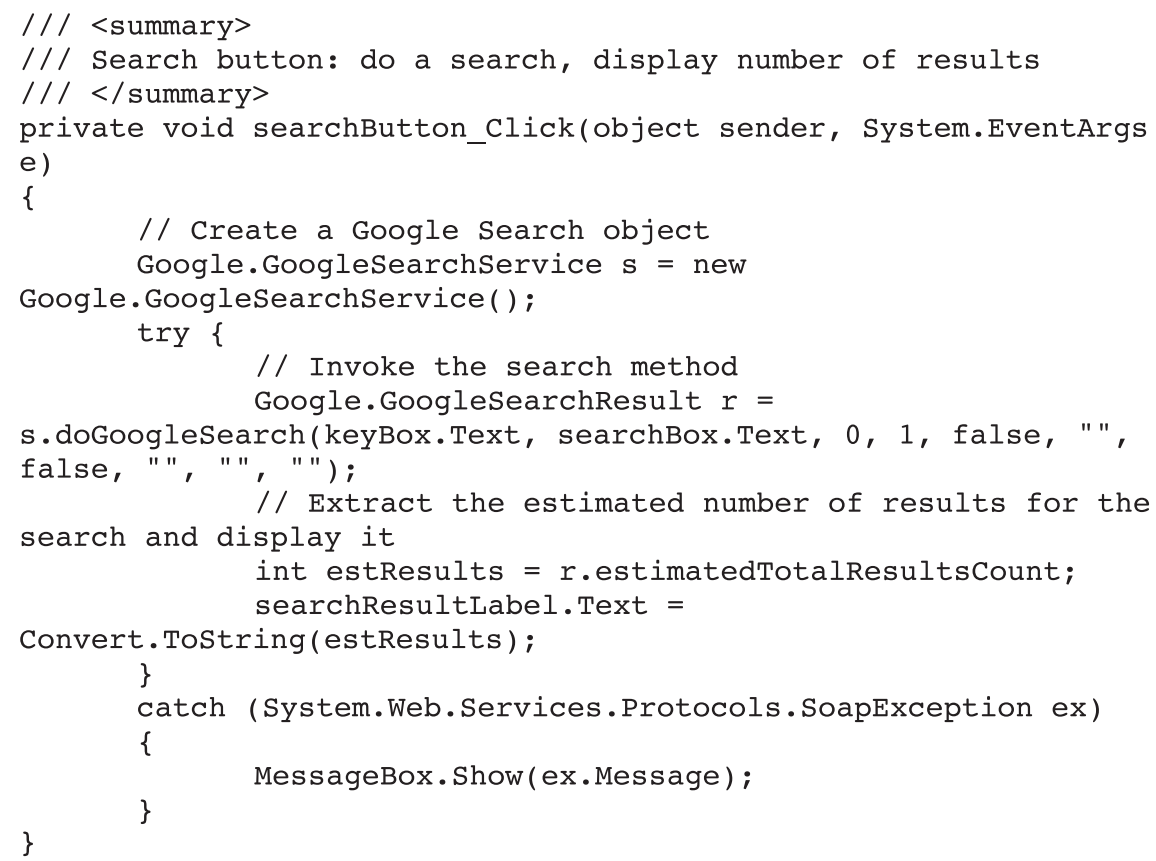

Figure 17

Source code of service requester application. 


\subsubsection{SOA}

In order to better integrate the concept of web services in enterprise systems, IT editors now propose the Service Oriented Architecture (SOA) approach (Sprott and Wilkes, 2004). Beyond the marketing hype, a consensus is established on the concept of service as an autonomous process which communicates by message within an architecture that identifies applications as services. This design is based on coarse-grained, loosely coupled services interconnected by asynchronous or synchronous communication and XML-based standards.

The definition and elements of SOA are not yet well established. Sessions (2003) wonders whether a SOA is:

- a collection of components over the Internet;

- the next release of CORBA or;

- an architecture for publishing and finding services.

Finally he suggests that a SOA is "an architecture that defines how autonomous systems interoperate with particular focus on asynchronous communications, heterogeneous transport channels, proof of identity, error management and workflow coordination".

A SOA is jusy an evolution of web distributed component based architectures to make applications integration easier, faster, cheaper and more flexible, improving return on investment. In fact the main innovations are in the massive adoption of web services (even if a SOA does not imply the use of a web services model and vice versa) by the industry and in the use of the XML language to describe services, processes, security and exchanges of messages, etc.This promises more future-proof IT projects than in the past.

Despite limitations of web services in fields such as early standards, security, orchestration, transactions, reliability, performance, and the ethic and economic model, the technology now appears to be complementary to solutions based on a classic middleware bus, as well as to EAI/ERP solutions. Its loose coupling brings increased flexibility and facilitates the re-use of legacy systems. Moreover web services can be used as low-cost connectors between distinct technological platforms such as COM, .NET and EJB. The next release of Microsoft's Windows operating system will include Indigo, a new interoperability technology based on web services, to unify Microsoft's proprietary communication mode. S. Abitboul, research director at INRIA, estimates that web services will represent, in the long run, the natural protocol for accessing information systems. Thus it seems that we are only at the start of web services and SOA.

\section{BRIDGING TECHNOLOGY}

Above we dealt with interoperability features within a particular inner technology. Bridging technology allows one to extend the aptitude of a system to interoperate between outer technologies. Two bridges are illustrated, given that SOAP and web services, as noted previously, may play the role of connector.

\subsection{COM and JAVA}

The use of COM components from the Java platform is not natural. Components of CORBA middleware can interact without considering the source code language. This is not obvious with COM and Java. Microsoft has proposed such functionalities in its implementation of Java with JactiveX tool, but this technology is abandoned because Microsoft Java implementation was not compliant with the language specification. One objective of the COGents project (Braunschweig et al., 2002), is to perform interactions between a multi-agent platform developed in Java and an INDISS modeling platform using JACOB components. JACOB is a Java-COM Bridge that allows COM Automation components to be called from Java. It uses Java Native Interface to make native calls into the COM and Win32 libraries. JACOB handles also COM events from a COM server to the Java client. Agents written in JAVA can send COM requests to the INDISS COM server. JACOB provides JAVA objects like "VARIANT" to be able to express COM calls. The JNI code of JACOB interacts with the COM server interface pointers to forward the call with $\mathrm{COM}$ parameters at the JNI level. This tool shows that the link between JAVA and COM exists, but it was not fully developed and tested because the code had to be corrected on switching from the JAVA virtual machine 1.3 to 1.4 .

\subsection{EJB and .NET}

With the growing popularity of .NET and the wide use of EJB, many actors of the middleware software development have tried to bridge the gap between these two technologies. Among the different solutions available we can mention Janeva from Borland, IIOP.NET from ELCA Informatique (2004), Remoting.CORBA (2004) and DotNetJ from ObjectWeb (2004). Peltzer (2003) examines the technical issues arising from integrating J2EE and .NET and offers practical solutions. Bonneau and Newcomer (2003) discuss SOAP and web services' promise to provide the best solution for bridging .NET and J2EE-based applications.

Basically, the different solutions provide tools to generate code from IDL and an IIOP engine used at run-time. They also provide native data type conversions between .NET built-in classes and J2EE classes or Java classes and viceversa. A clear description of Janeva can be found in Natarajan (2003).

\section{CONCLUSION}

Enterprises are characterized by an organization networked through their information system in which all the elements have to interact. This results in an increasing dependence with regard to information technologies for interoperability. Corresponding multi-tier architecture information systems 
are today built over advanced EJB or .NET component frameworks, themselves relying on middleware technologies such as CORBA, RMI and (D)COM. Initially EJB technology is multi-system and mono-language (Java) while .NET technology is mono-system (Windows) and multi-language. CCM aims at proposing a multi-system and multi-language technology. Solutions exist to "unlock" EJB and .NET. For example Common Language Infrastructure (CLI) and C\# programming language from .NET are now standardized by Ecma and ISO. Implementations on e.g. Linux are available.

In addition, web services and SOAP can give many benefits. Andrews (2004) predicts dramatic changes in the web services market for 2006, and announces a new class of business applications called "service-oriented business applications". The merging of web standards, IT and object/component technologies to form SOA and web services is announced as the next stage of evolution for e-business knowing that grid and autonomic computing should add their contributions too. There is no doubt that the scientific field will derive many benefits from this trend. The engineer already benefits from information technologies for interoperability, especially with XML, COM and CORBA using domain standards from POSC, OPC Foundation, CO-LaN, and domain applications such as OpenSpirit, INDISS. As illustrated by Sama et al. (2003) and Westhaus (2004) for the process engineering field, one can foresee many applications of SOA and web services in oil and gas sciences.

\section{REFERENCES}

Andrews, W. (2004) Predicts 2004, Gartner's Predictions. http://www3.gartner.com/research/spotlight/asset_55117_895.jsp

Arrieux, Y. (2003) Éditeurs et constructeurs veulent accélérer l'adoption des services web, dossier 01net : Electronic Business Days 2003 : l'entreprise interconnectée passée en revue, January. http://www.01net.com/article/200210.html

BearingPoint, SAP and Sun Microsystems (2003) Livre blanc, Les services web, Pourquoi ?

http://www.bearingpoint.fr/content/library/138_731.htm

Belaud, J.P. (2001) Introduction to the CAPE-OPEN standard and its related technology, CAPE-OPEN Update Journal, October, 1. www.colan.org

Belaud, J.P. and Pons, M. (2002) Open software architecture for process simulation. Computer-Aided Chemical Engineering, 10, May 2002, Elsevier, 847-852, ISBN: 0-444-51109-1.

Bloomberg, J. (2001) Web services: A New Paradigm for Distributed Computing, The Rational Edge, September. http://www-106.ibm.com/developerworks/rational/library/ content/RationalEdge/archives/sep01.html

Birrel, A.D. and Nelson, B.J. (1984) Implementing Remote Procedure Calls. ACM, Trans. on Computers Systems, 2, February, 39-59.

Boehm B. (2000) Spiral Development: Experience, Principles, and Refinements. Spiral Development Workshop, Ed. Wilfred J. Hansen, February. www.sei.cmu.edu/cbs/spiral2000/SR08.pdf

Bonneau R. and Newcomer E. (2003) Integrate .NET and J2EE with web services. Windows Server System Magazine, 3, 2. http://www.ftponline.com/wss/2003_02/magazine/features/rbonn eau/default.aspx
Booch, G., Rumbaugh, J. and Jacobson, I. (1998) Unified Modeling Language User Guide, Addison Wesley, ISBN: 0-20157168-4.

Box, D. (1998) Essential COM, Addison Wesly.

Braunschweig, B.L., Fraga, E.S., Guessoum Z., Paen, D., Piñol D. and Yang, A. (2002) COGents: a new IST-funded project on CAPE-OPEN software agents. CAPE-OPEN Update Journal, 4 , 17-21, November. www.colan.org

Brown, A.W. (1996) Component-Based Software Engineering: Selected Papers from the Software Engineering Institute, WileyIEEE Computer Society Press.

Browning, D. (2002) Integrate NET Remoting into the Enterprise. Windows Server System Magazine, 2,10. http://www.ftponline.com/wss/2002_11/magazine/features/dbrow ning/default.aspx

Chan, R. (2003) Adopting RUP in a COTS implementation project. The Rational Edge, May.

Eddon, G. and Eddon, H. (1998) Inside Distributed COM, Microsoft Press.

ELCA Informatique (2004) http://iiop-net.sourceforge.net/

Farley, J. (2000) Microsoft .NET vs. J2EE: How Do They Stack Up? January. http://java.oreilly.com/news/farley_0800.html

Fay, S. (2003) Standards and reuse. The Rational Edge, May. http://www-106.ibm.com/developerworks/rational/library/2277.html

Glass, G. (2001) How SOAP Works. The Web Services (R)evolution, Part 3.

http://www-106.ibm.com/developerworks/xml/library/ws-peer3/

Google Web APIs Beta (2004)

http://www.google.fr/apis/index.html

Gopalan, S.R. (2004) http://my.execpc.com/ gopalan/

Hofmeister, C., Nord, R. and Soni, D. (2000) Applied Software Architecture, Addison-Wesley Longman, Reading, MA.

Holloway R. (2002) Compare .NET Remoting to web services, Visual Studio magazine, Web services in the Enterprise, 12, 11. http://www.ftponline.com/vsm/2002_09_14th/online/holloway/de fault_pf.aspx

IBM developerWorks (2004) Web Services Category. http://www-136.ibm.com/developerworks/webservices/

IBM Glossary (2004) Glossary of Computing Terms. http://www306.ibm.com/ibm/terminology/goc/gocmain.htm

Koch, C. (2003) The Battle for web services. CIO Magazine, October. http://www.cio.com/archive/100103/standards.html

Linthicum, D.S. (2003) Next Generation Application Integration - From Simple Information to Web Services, September, Addison Wesley, ISBN: 0-201-84456-7.

Manes, A.T. (2003) Web Services A Manager's Guide, September, Addison Wesley, ISBN: 0-321-18577-3.

Meerkat (2000) O'Reilly Network's Open Wire Service. http://www.oreillynet.com/pub/a/rss/2000/11/14/meerkat_xmlrpc. html

Natarajan, V. (2004) .NET and J2EE integration. Technical Talk, January. http://www.theserverside.net/talks/index.aspx

Newcomer, E. (2002) Decide between J2EE and .NET web services. Windows Server System Magazine, $2,9$.

http://www.ftponline.com/wss/2002_10/magazine/columns/ webservices/

ObjectWeb Consortium (2004) www.objectweb.org

Oellermann, W. (2002) Create web services with business value. .NET Magazine, $\mathbf{2}, 10$.

http://www.ftponline.com/wss/2002_11/magazine/features/ wollermann/default.aspx 
OpenSpirit (2004) http://www.openspirit.com

Peltzer D. (2003) .Net \& J2EE Interoperability, November 2003, Osborne, ISBN: 0-072-23054-1

Puder A. (2004) http://www.puder.org/

Remoting.Corba (2004) http://remoting-corba.sourceforge.net/

Sama, S., Piñol, D. and Serra M. (2003), Web-based process engineering Petroleum Technology Quarterly, 2003.

Serain D. (2001) Entreprise Application Intégration L'architecture des solutions e-business, Avril 2001, Dunod, ISBN: 2-10-005605-0

Sessions R. (2000) Objects and Components, ObjectWatch newsletter number 28, June 2000,

http://www.objectwatch.com/issue_28.htm

Sessions R. (2003) What is a Service-Oriented Architecture (SOA)? ObjectWatch newsletter number 45, October 2003, http://www.objectwatch.com/issue_45.htm
Sprott D. and Wilkes L. (2004) Understanding Service Oriented Architecture, Microsoft Architects Journal, EMEA Edition, January 2004, http://www.thearchitectjournal.com/Journal/issue1/article2.html

TMC (2002) The Petstore Revisited: J2EE vs .NET Application Server Performance Benchmark, November 2002, http://www.middleware-company.com/j2eedotnetbench/

UserLand (2004) http://www.xml-rpc.com/

Wassermann A.I. (1990) Tool Integration in Software Engineering Environments, Software Engineering Environments, F. Long, Springer-Verlag, Berlin, 138-150.

Westhaus U. (2004) DETHERM...on the WEB, an online service from DECHEMA: http://i-systems.dechema.de/detherm/

XMLFAQ (2004) The XML FAQ,

http://www.ucc.ie:8080/cocoon/Xmlfaq

Final manuscript received in October 2004 